\title{
Background Risk and the Performance of Insurance Markets under Adverse Selection
}

\author{
Keith J. Crocker ${ }^{\mathrm{a}}$ and Arthur Snow ${ }^{\mathrm{b}}$ \\ ${ }^{a}$ Smeal College of Business, Pennsylvania State University, University Park, PA 16802, U.S.A. \\ ${ }^{\mathrm{b}}$ Department of Economics, University of Georgia, Athens, GA 30602, U.S.A. \\ E-mail: snow@terry.uga.edu
}

Background risk can influence the performance of insurance markets that must deal with adverse selection when applicants are risk vulnerable, since they are more averse to bearing the insurable risk as a result of their exposures to background risk. We show that background risk always results in a lower deductible for the incentive constrained contract, and that a broader range of markets attains the stable sequential equilibrium cross-subsidized pair of separating contracts. We conclude that background risk always improves the performance of markets for coverage against (insurable) foreground risks that must deal with adverse selection. We also find, however, that these improvements are never sufficient to offset the cost to insureds of bearing the background risk.

The Geneva Risk and Insurance Review (2008) 33, 137-160. doi:10.1057/grir.2008.12

Keywords: Nash screening equilibrium; risk vulnerability; prudence

\section{Introduction}

The performance of insurance markets can be affected by the presence of background risks, such as those associated with uninsurable fluctuations in the value of human capital arising from randomness in entrepreneurial and wage incomes. For individuals who are risk vulnerable in the sense defined by Gollier and Pratt (1996), exposure to fair or unfair background risk increases the degree of aversion toward bearing the foreground, insurable risks and, as a result, alters the demand for insurance against these risks. This would be of no consequence if full and fair insurance were available to all individuals. ${ }^{1}$ However, in the presence of adverse selection, insurance contracts must satisfy incentive compatibility constraints that result in partial coverage for some

${ }^{1}$ Eeckhoudt and Kimball (1992) show that, with proportional loading in the insurance premium, an individual demands more coverage when exposed to fair background risk if absolute risk aversion and absolute prudence are both decreasing functions of wealth. Gollier and Pratt show that such an individual is risk vulnerable with respect to the introduction of fair or unfair background risk. 
insurance applicants. Changes in the degree of risk aversion alter these incentive constraints and, in addition, affect the attractiveness of competing contractual offers. Thus, background risk can influence the performance of insurance markets through either of two channels that bear directly on the market equilibrium attained under adverse selection.

Following Rothschild and Stiglitz (1976) and Hellwig (1987), we assume that competitive insurers, unable to distinguish among applicants by their risk of incurring a given loss, deal with adverse selection by engaging in price-quantity competition. We relax their assumption that each insurer can offer only one type of contract, and instead assume that insurers can offer applicants the opportunity to choose from a menu of contracts that incorporate different combinations of premium and deductible. The market attains a Nash screening equilibrium that depends on the proportion of high risks in the applicant pool. If this proportion is greater than or equal to a critical value, then the market attains the pure strategy Nash equilibrium identified by Rothschild and Stiglitz, and offers applicants a separating pair of contracts that breakeven individually; otherwise, the market attains a sequential screening equilibrium and offers the cross-subsidized pair of separating contracts identified by Miyazaki (1977).

We show that exposure to background risk causes this critical proportion of high-risk applicants to increase, so that the cross-subsidized equilibrium is attained over a broader range of insurance markets. Since the cross-subsidized contracts provide greater coverage of the foreground risk than that afforded by the breakeven contracts, background risk improves the performance of the insurance markets when it causes the equilibrium to switch from the breakeven pair to a cross-subsidized pair. Additionally, even when the nature of the market equilibrium is not affected by the presence of background risk, the incentive constraints are relaxed and the market provides greater coverage of the foreground risk. Thus, background risk always enhances the performance of insurance markets under adverse selection.

It does not follow, however, that the presence of background risks is desirable, since these risks are unavoidable and costly to bear. Indeed, we find that insurance applicants are always worse off when background risk is present despite the improved performance of markets insuring the foreground risk. We conclude that public and private innovations that limit exposure to background risks or extend insurance coverage to them would improve the welfare of all insurance applicants.

Several previous studies of adverse selection in insurance markets have drawn attention to radical changes in the contracting environment that are introduced when the degree of risk aversion is hidden knowledge. Arnott and Stiglitz (1988) explore the desirability of introducing ex post randomization, Landsberger and Meilijson (1994, 1999) consider monopolistic contracts, while 
Smart (2000) and Wambach (2000) investigate the implications of "double crossing" indifference curves that can occur when insurance applicants differ with respect to their hidden knowledge of both risk and risk aversion. We, in contrast, focus on situations in which insurers can categorize applicants on the basis of observable characteristics correlated with risk aversion and effectively control for any differences in applicants' willingness to bear insurable risk, so that their degree of risk aversion is not hidden knowledge. Moreover, in our analysis, differences in risk aversion are not immutable, but instead arise from the presence of background risks. Our model thus offers insights regarding the potential social value of innovations that extend coverage to previously uninsurable (background) risks.

In the next section, we set out the model insurance market with adverse selection and illustrate the alternative equilibrium configurations. In the third section, we show that, when the risk preferences of insurance applicants are characterized by nonincreasing absolute risk aversion, an increase in risk aversion increases the critical proportion of high risks, so that the Rothschild-Stiglitz equilibrium is sustained over a narrower range of markets. In the fourth section, we introduce background risk as the cause of the increased aversion to bearing the insurable foreground risk and analyze its effect on the performance of the insurance market and the equilibrium welfare of risk vulnerable insurance applicants. Conclusions are presented in the last section.

\section{An insurance market with adverse selection}

As in the Rothschild-Stiglitz model, we assume that applicants for insurance are endowed with an initial wealth $W$, a strictly risk-averse von NeumannMorgenstern utility function $U$, and incur a loss $D$ with probability $p^{H}$ or the smaller probability $p^{L}$. Each applicant's loss probability is hidden knowledge, while insurers know the magnitude of the potential loss, the two loss probabilities, and the proportion of high-risk applicants in the population, denoted by $\lambda$. An insurance contract specifies a premium and a deductible, but it will be useful to represent contracts in terms of the state-contingent wealth they provide to an insurance applicant. To this end, let $W_{N}$ denote wealth for the state in which the loss is not incurred, implying a premium of $W-W_{N}$, and let $W_{A}$ denote wealth for the accident state when the loss is incurred, implying a deductible of $W_{N}-W_{A}$. When purchased by a $t$-type applicant, the contract's expected profit is the premium collected minus the expected indemnity, $\left(W-W_{N}\right)-p^{t}\left[D-\left(W_{N}-W_{A}\right)\right]$, which may be written as

$$
\left(W-p^{t} D\right)-\left[\left(1-p^{t}\right) W_{N}+p^{t} W_{A}\right] .
$$


140

Thus, the expected profit of the contract is the applicant's expected wealth less the expected wealth provided by the contract. In addition, the applicant realizes the expected utility $\left(1-p^{t}\right) U\left(W_{N}\right)+p^{t} U\left(W_{A}\right)$.

We assume that each insurer attracts a pool of applicants that is representative of the population as a whole and deals with the adverse selection engendered by the hidden knowledge of risk type by offering a menu of contracts from among which applicants choose their most preferred option. Following Hellwig (1987), we assume that insurers may then refuse to honor the choices made by the applicants by rejecting all applicants to each of its contract offers and exiting the market. The equilibrium for this contracting environment depends on the proportion $\lambda$ of high-risks in the population. When $\lambda$ is greater than or equal to a critical value $\lambda^{*}$, the equilibrium is the separating pair of contracts identified by Rothschild and Stiglitz as the pure strategy Nash screening equilibrium. These contracts break even individually and are incentive compatible, so that an applicant of type $t$ prefers the contract intended for risk class $t$. Competition for $H$-types leads to full and fair insurance for these applicants whose insured wealth is $W^{H}=W-p^{H} D$, while competition for $L$-types minimizes the cost they bear from the adverse selection externality by incorporating the smallest deductible consistent with incentive compatibility and the requirement that each contract break even individually. As a result, the only binding incentive constraint is the one for $H$-types, requiring

$$
U\left(W^{H}\right)=\left(1-p^{H}\right) U\left(W_{N}^{L}\right)+p^{H} U\left(W_{A}^{L}\right),
$$

where $\left(W_{N}^{L}, W_{A}^{L}\right)$ is the partially insured wealth position of the $L$-types that yield an expected profit of zero in expression (1) while satisfying Eq. (2). We adopt the standard assumption that $H$-types choose their full insurance contract as it offers more coverage. We shall refer to these as the "RS contracts."

Figure 1 illustrates the contingent wealth positions that result from these contracts, with the $H$-types receiving full and fair insurance at $H^{*}$, and the $L$-types bearing a deductible at $L$ where the $H$-type indifference curve labeled $U^{H}$ cuts the $L$-type fair-odds line passing from $E$ through $L$, along which $L$-type contracts earn zero profit. The RS contracts constitute a pure strategy Nash screening equilibrium if there is no alternative pair of contracts that can attract applicants away from these contracts and earn positive profit. This is the situation illustrated in Figure 1. Any point $\left(\hat{W}_{N}^{L}, \hat{W}_{A}^{L}\right)$ on the locus $F L$ results from an $L$-type contract that pairs with a fully insured wealth $\hat{W}^{H}$ for $H$-types to satisfy the incentive constraint (2) as well as the zero-profit condition

$$
\begin{aligned}
& (1-\lambda)\left[\left(1-p^{L}\right) \hat{W}_{N}^{L}+p^{L} \hat{W}_{A}^{L}\right]+\lambda \hat{W}^{H} \\
& \quad=W-\left[(1-\lambda) p^{L}+\lambda p^{H}\right] D,
\end{aligned}
$$




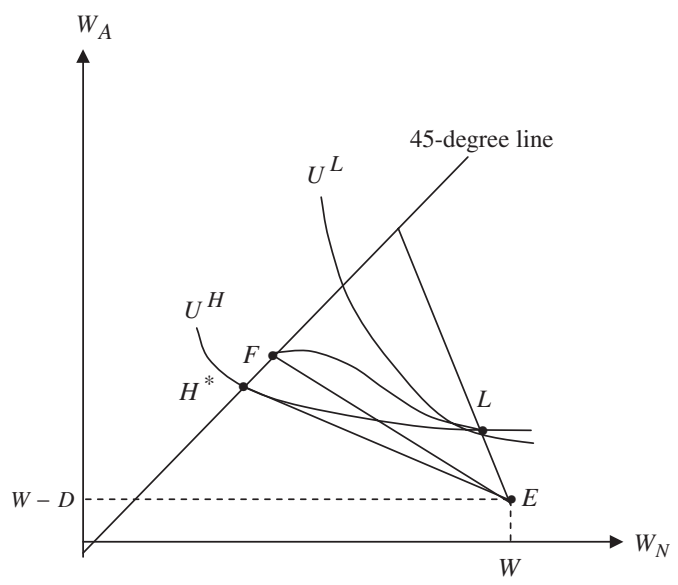

Figure 1. $\lambda=\lambda^{*}$

which allows for cross-subsidization between the two contracts. As $\lambda$ declines, the slope of the locus $F L$ increases. Figure 1 depicts the case in which $\lambda=\lambda^{*}$ and the RS contracts are just about to fail as an equilibrium. When $\lambda$ falls below $\lambda^{*}$, the locus $F L$ rises above the $L$-type indifference curve, and there exist profitable, Pareto preferred defections from the RS contracts. As a consequence, the RS contracts cannot be sustained as a Nash equilibrium.

To address cases in which the RS contracts are unsustainable, Hellwig, elaborating on the approach taken by Wilson (1977), examines a three-stage game in which insurers make contract offers in the first stage and in the third stage can reject applicants choosing their offers in the second stage. Hellwig shows that, when the RS contracts are unsustainable, the strategically stable sequential equilibrium is the pooling contract most preferred by $L$-types among those that satisfy the zero-profit condition (3), provided each insurer is restricted to offering one type of contract. More realistically, insures can offer more than one type of contract, and in that event the sequential equilibrium is the cross-subsidized separating pair most preferred by $L$-types among those

\footnotetext{
${ }^{2}$ By assuming that each insurer can offer only one type of contract, Rothschild and Stiglitz focus on pooling contracts as potentially profitable defections. When this assumption is relaxed and insurers can offer more than one contract, pairs of contracts in which losses generated by the $H$-type contract are offset by profits earned on the $L$-type contract are also potentially profitable defections, as discussed by Rothschild and Stiglitz. Profitable defections of this type do not require as small a value for $\lambda$ as is required for pooling defections to be profitable, a point established in Crocker and Snow (1985a).
} 


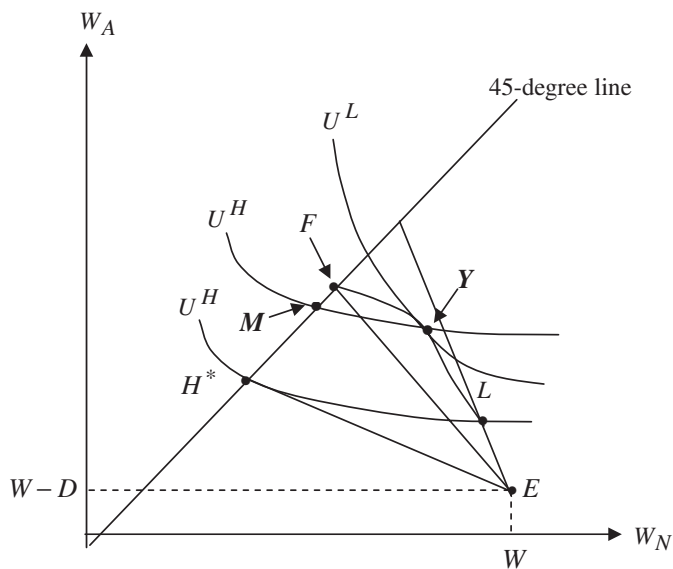

Figure 2. $\lambda<\lambda^{*}$

satisfying the incentive constraint (2) and the zero-profit condition (3) when, at the third stage, each insurer must either accept or reject all applicants to all of its offers. ${ }^{3}$ This outcome, identified by Miyazaki and analyzed by Spence (1978), referred to as the Wilson-Miyazaki-Spence (WMS) outcome, is illustrated in Figure 2 as the contingent wealth positions $M$, providing full insurance for $H$-types, and $Y$, offering partial coverage to $L$-types along locus $F L{ }^{4}$

Thus, the nature of equilibrium depends critically on the proportion of $H$-types. When this proportion is at least as great as $\lambda^{*}$, the $\mathrm{RS}$ contracts

${ }^{3}$ When insurers are restricted to making single contract offers, rejecting all applicants at the third stage is equivalent to exiting from the market. Extending this rejection strategy to environments in which each insurer can offer more than one type of contract results in the WMS outcome. If, instead, an insurer can reject applicants to some of its offers while accepting applicants to its remaining offers, then the WMS outcome cannot be sustained, as an insurer would reject applicants to the high-risk contract on which it expects to make a loss, leading to the WilsonHellwig pooling outcome. Asheim and Nilssen (1996) suggest an alternative third stage in which each insurer can renegotiate exclusively with all its own applicants, and they conclude that the unique equilibrium is the WMS outcome.

${ }^{4}$ One complicating factor, which was noted by Miyazaki, is that there may be more than one solution to the problem of maximizing the expected utility of the $L$-types subject to constraints (2) and (3) because of non-convexities in the choice set. We have assumed that when $H$-types are indifferent between two contracts they choose the one offering more coverage. Extending this assumption to the $L$-types resolves the non-uniqueness problem and ensures that the equilibrium is second best efficient. 
constitute an equilibrium, while otherwise we assume the equilibrium is the Pareto preferred WMS outcome. ${ }^{5}$ As a prelude to studying the implications of introducing background risk, we first examine the effect on $\lambda^{*}$ of increasing the insurance applicants' degree of risk aversion.

\section{The effect of greater risk aversion on insurance markets with adverse selection}

We are interested in the effect of background risk on the performance of the insurance market set out in the previous section when the utility function $U$ is risk vulnerable, so that applicants are more averse to bearing the foreground risk as a result of their exposures to background risks. Accordingly, in this section we examine the effect of increasing the degree of risk aversion on the nature of equilibrium in the insurance market. Specifically, we identify conditions under which the critical value $\lambda^{*}$ increases as the utility function $U$ becomes more risk averse.

An increase in risk aversion has no effect on the RS contract for $H$-types, since competition ensures that they receive full and fair insurance. However, they have a smaller acceptance set, which relaxes the incentive constraint and results in a lower deductible in the $L$-type contract. This "deductible" effect makes it more difficult to attract $L$-types to profitable defections from their RS contract. However, there is a countervailing effect as the $L$-types also have a smaller acceptance set, so their indifference curves are less steeply sloped at their RS contingent wealth position. This "risk aversion" effect makes it easier to attract them to a defecting contract. Thus, as risk aversion increases the effect on $\lambda^{*}$ is ambiguous in general. However, the following result shows that the risk aversion effect dominates, and $\lambda^{*}$ increases, when $U$ exhibits nonincreasing absolute risk aversion.

Proposition 1 When the utility function $U$ becomes more risk averse, the critical value $\lambda^{*}$ increases if $U$ exhibits nonincreasing absolute risk aversion.

Proof To establish this result, we first derive an expression for $\lambda^{*}$ by equating the $L$-type marginal rate of substitution with the slope of locus $F L$ at the $L$-type contingent wealth $L=\left(W_{N}^{L}, W_{A}^{L}\right)$ attained under the RS contracts. We then show that $\lambda^{*}$ decreases as a result of the deductible effect, but

\footnotetext{
${ }^{5}$ Our assumption that equilibrium is the RS outcome when $\lambda \geqslant \lambda^{*}$ and is otherwise the WMS outcome ensures that the market always attains a second-best efficient allocation given the adverse selection externality as shown by Crocker and Snow (1985a). Thus, when analyzing the welfare implications of background risk, we are assured that there is no possibility of improving on the market's performance.
} 
increases as a result of the risk aversion effect. In Lemma 1, we show that the latter dominates the former, so that $\lambda^{*}$ increases, when $U$ belongs to the class of functions that exhibit constant absolute risk aversion (CARA). Then in Lemma 2, we show that the deductible effect is smaller when $U$ exhibits decreasing absolute risk aversion (DARA) rather than CARA. The Proposition then follows from these two Lemmas.

Let $W^{H}=W-p^{H} D$ denote the wealth of an $H$-type under their RS contract. To derive the slope of locus $F L$, differentiate the incentive constraint (2), solve for $d W^{H}$, substitute the result into the differential equation implied by the zero-profit condition (3), and rearrange terms to obtain

$$
-\frac{d W_{A}^{L}}{d W_{N}^{L}}=\frac{\lambda\left(1-p^{H}\right) U_{N}^{\prime}+(1-\lambda)\left(1-p^{L}\right) U_{H}^{\prime}}{\lambda p^{H} U_{A}^{\prime}+(1-\lambda) p^{L} U_{H}^{\prime}}
$$

as the slope of locus $F L$ at the $L$-type RS contract. Note that primes denote derivatives and the subscripts indicate the wealth argument. By setting $\lambda=\lambda^{*}$ in Eq. (4), equating the right-hand side to the $L$-type marginal rate of substitution at the $L$-type contract, and then solving the resulting equation for $\lambda^{*}$, we arrive at $\lambda^{*}=X /(P+X)$, where $P=\left(p^{H}-p^{L}\right) / p^{L}\left(1-p^{L}\right)$ and

$$
X=\frac{U_{H}^{\prime}}{U_{N}^{\prime}}-\frac{U_{H}^{\prime}}{U_{A}^{\prime}} .
$$

It follows that an increase in risk aversion increases $\lambda^{*}$ if and only if it increases $X$.

Let $\rho$ denote the index of absolute risk aversion for $U$. The deductible effect associated with an increase in $\rho$ entails an increase in $W_{A}^{L}$ and a reduction in $W_{N}^{L}$ as the $L$-type contract moves up their fair-odds line when the $H$-types become more risk averse. The zero-profit condition for the $L$-type contract implies

$$
\frac{d W_{N}^{L}}{d \rho}=-\frac{p^{L}}{1-p^{L}} \frac{d W_{A}^{L}}{d \rho}<0 .
$$

Using this relation, the total effect on $X$ of an incremental increase in risk aversion can be written as

$$
\frac{d X}{d \rho}=\frac{\partial X}{\partial \rho}+\left[\frac{U_{H}^{\prime}}{U_{N}^{\prime 2}} U_{N}^{\prime \prime} \frac{p^{L}}{1-p^{L}}+\frac{U_{H}^{\prime}}{U_{A}^{\prime 2}} U_{A}^{\prime \prime}\right] \frac{d W_{A}^{L}}{d \rho},
$$

where $\partial X / \partial \rho$ is the risk aversion effect (i.e., the direct effect of $\rho$ on $X$ through the function $U$ ) and the remaining terms on the right-hand side comprise the deductible effect (the indirect effect of $\rho$ on $X$ through the wealth arguments of $U$ ), which is clearly negative.

To investigate the risk aversion effect, recall the fundamental result of Pratt (1964) showing that an increase in risk aversion for $U$ is equivalent to an increasing, strictly concave transformation of $U$. Accordingly, assume that 
$G(\cdot)$ is increasing and strictly concave, and replace $U$ with $G(U)$. Then, in the expression for $X, G^{\prime}(U) U^{\prime}$ replaces $U^{\prime}$. Since $W_{N}^{L}>W^{H}>W_{A}^{L}$ and $G$ is strictly concave, we have $G^{\prime}\left(U\left(W_{N}^{L}\right)\right)<G^{\prime}\left(U\left(W^{H}\right)\right)<G^{\prime}\left(U\left(W_{A}^{L}\right)\right)$. As a result, we have

$$
\frac{G^{\prime}\left(U\left(W^{H}\right)\right) U_{H}^{\prime}}{G^{\prime}\left(U\left(W_{N}^{L}\right)\right) U_{N}^{\prime}}>\frac{U_{H}^{\prime}}{U_{N}^{\prime}} \text { and } \frac{G^{\prime}\left(U\left(W^{H}\right)\right) U_{H}^{\prime}}{G^{\prime}\left(U\left(W_{A}^{L}\right)\right) U_{A}^{\prime}}<\frac{U_{H}^{\prime}}{U_{A}^{\prime}} .
$$

Together, these inequalities imply

$$
\frac{G^{\prime}\left(U\left(W^{H}\right)\right) U_{H}^{\prime}}{G^{\prime}\left(U\left(W_{N}^{L}\right)\right) U_{N}^{\prime}}-\frac{G^{\prime}\left(U\left(W^{H}\right)\right) U_{H}^{\prime}}{G^{\prime}\left(U\left(W_{A}^{L}\right)\right) U_{A}^{\prime}}>\frac{U_{H}^{\prime}}{U_{N}^{\prime}}-\frac{U_{H}^{\prime}}{U_{A}^{\prime}},
$$

indicating that $\partial X / \partial \rho$ is positive.

Thus, the risk aversion effect causes $\lambda^{*}$ to increase, while the deductible effect works in the opposite direction. We establish the result stated in the Proposition with the aid of two Lemmas. The first shows that the risk aversion effect dominates when $U$ exhibits CARA.

Lemma 1 When utility belongs to the family of CARA functions, an increase in risk aversion increases $\lambda^{*}$.

Proof of Lemma 1 Let $U(W)=-\exp [-\rho W]$, so that the index of absolute risk aversion is constant and equal to $\rho$. Using this utility function to evaluate (5), we have

$$
X=\exp \left[\rho\left(W_{N}^{L}-W^{H}\right)\right]-\exp \left[\rho\left(W_{A}^{L}-W^{H}\right)\right],
$$

and the total effect on $X$ of an increase in $\rho$ is given by

$$
\begin{aligned}
\frac{d X}{d \rho}= & \exp \left[\rho\left(W_{N}^{L}-W^{H}\right)\right]\left[\rho \frac{d W_{N}^{L}}{d \rho}+W_{N}^{L}-W^{H}\right] \\
& +\exp \left[\rho\left(W_{A}^{L}-W^{H}\right)\right]\left[-\rho \frac{d W_{A}^{L}}{d \rho}+W^{H}-W_{A}^{L}\right] .
\end{aligned}
$$

Diffferentiating the binding incentive constraint (2) for $H$-types with respect to $\rho$ yields

$$
\begin{aligned}
W^{H} \exp \left[-\rho W^{H}\right]= & W_{N}^{L}\left(1-p^{H}\right) \exp \left[-\rho W_{N}^{L}\right] \\
& +W_{A}^{L} p^{H} \exp \left[-\rho W_{A}^{L}\right] \\
& +\left(1-p^{H}\right) \exp \left[-\rho W_{N}^{L}\right] \rho \frac{d W_{N}^{L}}{d \rho} \\
& +p^{H} \exp \left[-\rho W_{A}^{L}\right] \rho \frac{d W_{A}^{L}}{d \rho}
\end{aligned}
$$


After substituting for $\exp \left[-\rho W^{H}\right]$ from the $H$-type incentive constraint (2), Eq. (12) can be rewritten as

$$
\begin{aligned}
& \left(1-p^{H}\right) \exp \left[-\rho W_{N}^{L}\right]\left[\rho \frac{d W_{N}^{L}}{d \rho}+W_{N}^{L}-W^{H}\right] \\
& \quad=p^{H} \exp \left[-\rho W_{A}^{L}\right]\left[-\rho \frac{d W_{A}^{L}}{d \rho}+W^{H}-W_{A}^{L}\right] .
\end{aligned}
$$

Observe that the two bracketed terms in Eq. (13) must have the same sign, and that Eq. (11) implies $d X / d \rho$ is positive if these terms are positive. Using Eq. (6) to account for the zero-profit condition for the $L$-type contract, we solve (13) for $\rho d W_{A}^{L} / d \rho$, multiply the result by $-p^{L} /\left(1-p^{L}\right)$, add $W_{N}^{L}-W^{H}$, and rearrange terms to arrive at

$$
\begin{aligned}
- & \rho \frac{p^{L}}{1-p^{L}} \frac{d W_{A}^{L}}{d \rho}+W_{N}^{L}-W^{H} \\
& =\frac{p^{H} \exp \left[-\rho W_{A}^{L}\right]\left\{W^{H}-\left[\left(1-p^{L}\right) W_{N}^{L}+p^{L} W_{A}^{L}\right]\right\}}{p^{L}\left(1-p^{H}\right) \exp \left[-\rho W_{N}^{L}\right]-\left(1-p^{L}\right) p^{H} \exp \left[-\rho W_{A}^{L}\right]} .
\end{aligned}
$$

Since $p^{L}\left(1-p^{H}\right)<p^{H}\left(1-p^{L}\right)$ and $W_{N}^{L}>W_{A}^{L}$, the denominator is negative. Further, since both the $H$-type and $L$-type contracts breakeven individually, the term in braces in the numerator reduces to $\left(p^{L}-p^{H}\right) D$, which is negative. As a result, both the numerator and denominator on the right-hand side of Eq. (14) are negative, so the expression is positive and, therefore, $d X / d \rho$ is positive.

The second Lemma shows that the deductible effect is smaller when $U$ exhibits DARA rather than CARA.

Lemma 2 With an increase in risk aversion, the decline in the deductible in the $L$-type RS contract is smaller when utility belongs to the family of DARA functions than it is when utility belongs to the family of CARA functions.

Proof of Lemma 2 Consider an incremental increase in risk aversion. Let $V$ denote the resulting utility function, and let $\left(W_{N}^{L}, W_{A}^{L}\right)$ denote the $L$-type contingent wealth under the associated RS contract. Let $\bar{W}=\left(1-p^{H}\right) W_{N}^{L}+$ $p^{H} W_{A}^{L}$ denote the expected value of the $L$-type contingent wealth, but evaluated using $p^{H}$ as the probability of accident. Then the risk premium for $V$, denoted by $\pi$, is defined implicitly by

$$
V(\bar{W}-\pi)=\left(1-p^{H}\right) V\left(W_{N}^{L}\right)+p^{H} V\left(W_{A}^{L}\right) .
$$

Since the incentive constraint (2) must hold at the RS contracts, the argument on the left-hand side of (15) equals $W^{H}$ for any utility function, and $W^{H}$ is the 
result of full and fair insurance for $H$-types, which does not depend on the particular utility function being considered.

As $V$ is incrementally more risk averse than $U$, the risk premium for $V$ is larger than that for $U$. However, since $\bar{W}-\pi=W^{H}$ for any utility function, $\bar{W}$ must increase as the risk premium increases. It is straightforward to show that an increase in $\bar{W}$, and the requirement that $\left(W_{N}^{A}, W_{A}^{N}\right)$ earns zero profit from $L$-types, results in a lower deductible for $L$-types. We will show that the increase in $\bar{W}$ leads to a smaller reduction in the deductible when $V$ exhibits DARA rather than CARA.

Consider first an incremental increase in $\bar{W}$, and let $d W$ be an increase in the wealth of $L$-types in both states that maintains the equality at (15). We then have

$$
V^{\prime}(\bar{W}-\pi) d \bar{W}=\left[\left(1-p^{H}\right) V^{\prime}\left(W_{N}^{L}\right)+p^{H} V^{\prime}\left(W_{A}^{L}\right)\right] d W .
$$

The right-hand side of (16) may be written as $V^{\prime}(\bar{W}-\psi) d W$, where $\psi$ is the prudence premium introduced by Kimball (1990). Hence, the rate at which $W$ increases as $\bar{W}$ increases is given by

$$
\frac{d W}{d \bar{W}}=\frac{V^{\prime}(\bar{W}-\pi)}{V^{\prime}(\bar{W}-\psi)},
$$

which implies that the increase in $W$ is less than (equal to) the increase in $\bar{W}$ if $\pi$ is less than (equal to) $\psi$. To examine the relationship between $\pi$ and $\psi$, rewrite Eq. (15) as

$$
V(\bar{W}-\pi)=E[V(\bar{W}+\tilde{x})],
$$

where $E$ is the expectation operator and $\tilde{x}$ is an appropriately specified random variable, ${ }^{6}$ and consider the effect on $\pi$ of an increase in $\bar{W}$ with the random variable $\tilde{x}$ unchanged. Differentiating Eq. (18) with respect to $\bar{W}$ and solving for the effect on $\pi$ we obtain

$$
\begin{aligned}
\frac{d \pi}{d \bar{W}}= & \frac{V^{\prime}(\bar{W}-\pi)-E\left[V^{\prime}(\bar{W}+\tilde{x})\right]}{V^{\prime}(\bar{W}-\pi)} \\
& =\frac{V^{\prime}(\bar{W}-\pi)-V^{\prime}(\bar{W}-\psi)}{V^{\prime}(\bar{W}-\pi)} .
\end{aligned}
$$

The left-hand side of this expression is, by definition, negative (zero) when $V$ exhibits DARA (CARA), which implies that $\pi$ is less than (equal to) $\psi$. Thus, we have $d W / d \bar{W}<(=) 1$ as $V$ exhibits DARA (CARA).

${ }^{6}$ Specifically, $\tilde{x}$ takes the value $W_{N}^{L}-\bar{W}=p^{H}\left(W_{N}^{L}-W_{A}^{L}\right)$ with probability $1-p^{H}$, and takes the value $W_{A}^{L}-\bar{W}=\left(1-p^{H}\right)\left(W_{A}^{L}-W_{N}^{L}\right)$ with probability $p^{H}$. 
We complete the proof of Lemma 2 by relating the increase in $W$ to the reduction in the $L$-type deductible. Along the $L$-type fair-odds line, zero profit requires that $W_{N}^{L}=\left(p^{L} W_{A}^{L}+W-p^{L} D\right) /\left(1-p^{L}\right)$. Substituting this into the expected utility $\left(1-p^{H}\right) V\left(W_{N}^{L}\right)+p^{H} V\left(W_{A}^{L}\right)$ and differentiating with respect to $W_{A}^{L}$ yields the change in expected utility as $W_{A}^{L}$ increases (and, as the deductible declines) along the $L$-type fair odds line. Equating this change in expected utility with the right-hand side of Eq. (16), which gives the change in expected utility as $W$ increases, yields

$$
\begin{gathered}
{\left[-\frac{p^{L}}{1-p^{L}}\left(1-p^{H}\right) V_{N}^{\prime}+p^{H} V_{A}^{\prime}\right] \frac{d W_{A}^{L}}{d \bar{W}}} \\
=\left[\left(1-p^{H}\right) V_{N}^{\prime}+p^{H} V_{A}^{\prime}\right] \frac{d W}{d \bar{W}} .
\end{gathered}
$$

With DARA (CARA), we have $d W / d \bar{W}<(=) 1$, so the right-hand side of (20) is smaller with DARA than with CARA and, therefore, $d W_{A}^{L} / d \bar{W}$ is also smaller with DARA than with CARA. It follows that the decline in the $L$-type deductible is smaller with DARA than it is with CARA.

To complete the proof of the Proposition, let $G(\cdot)$ be a strictly concave function such that $V=G(U)$ is incrementally more risk averse than $U$. We have established that the risk aversion effect in Eq. (7) is positive and, in Lemma 1, that this effect dominates the negative deductible effect when $U$ exhibits CARA. As shown in Lemma 2, the risk aversion effect continues to dominate when $U$ exhibits DARA, since $d W_{A}^{L} / d \rho$ is smaller with DARA than it is with CARA. Since, with CARA, $\lambda^{*}$ increases because the risk aversion effect dominates the countervailing deductible effect, and the deductible effect is smaller with DARA, $\lambda^{*}$ must increase with any increase in risk aversion when $U$ exhibits nonincreasing absolute risk aversion.

Regarding Lemma 1, which refers to the case of CARA, Eq. (11) can be rewritten as

$$
\begin{aligned}
\frac{d X}{d \rho}= & \left\{\exp \left[\rho\left(W_{A}^{L}-W^{H}\right)\right]-\exp \left[\rho\left(W_{N}^{L}-W^{H}\right)\right\}\right. \\
& \times\left\{-\rho \frac{d W_{A}^{L}}{d \rho}+W^{H}-W_{A}^{L}\right\}+\exp \left[\rho\left(W_{N}^{L}-W^{H}\right)\right] \\
& \times\left\{\rho \frac{d\left(W_{N}^{L}-W_{A}^{L}\right)}{d \rho}+W_{N}^{L}-W_{A}^{L}\right\} .
\end{aligned}
$$

The first term within braces is negative, since $W_{A}^{L}$ is less than $W_{N}^{L}$, and we establish in the proof that the second term within braces is positive. Since we 


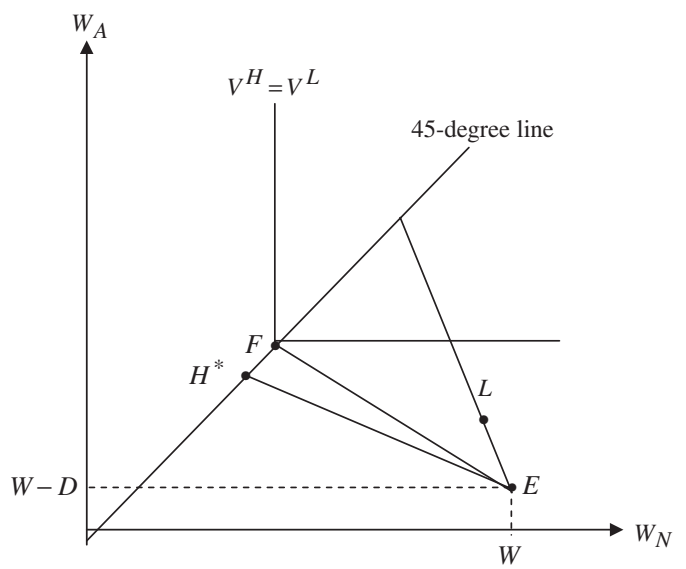

Figure 3. Extreme risk aversion

also establish that $d X / d \rho$ is positive, it must be the case that the final term within braces is also positive. Hence, we must have

$$
\frac{d \rho}{\rho}>-\frac{d\left(W_{N}^{L}-W_{A}^{L}\right)}{W_{N}^{L}-W_{A}^{L}},
$$

indicating that the proportional rate of increase in the degree of risk aversion exceeds the rate at which the deductible declines. Intuition for this inequality relation is provided by the extreme case in which risk aversion becomes infinite, illustrated in Figure 3. Risk aversion has increased to its limit, hence the Leontief-type indifference curve, yet a substantial deductible remains. ${ }^{7}$ Lemma 1 shows that this is the result of inequality (22).

Lemma 2 is illustrated in Figure 4 . The solid $H$-type indifference curve is associated with the incentive constraint (2) for utility function $U$. The lower of the two dotted indifference curves is associated with the indifference relation (15) for the more risk-averse utility function $V$, and illustrates the fact that an increase in risk aversion causes the $H$-type indifference curve to become flatter at $L$ leading to an intersection with the 45-degree line below the fully

${ }^{7}$ To ensure that $L$-types take the contract with the deductible, consider a case arbitrarily close to the limit of infinite risk aversion, so that the $L$-type indifference curves are still steeper than those of an $H$-type. 


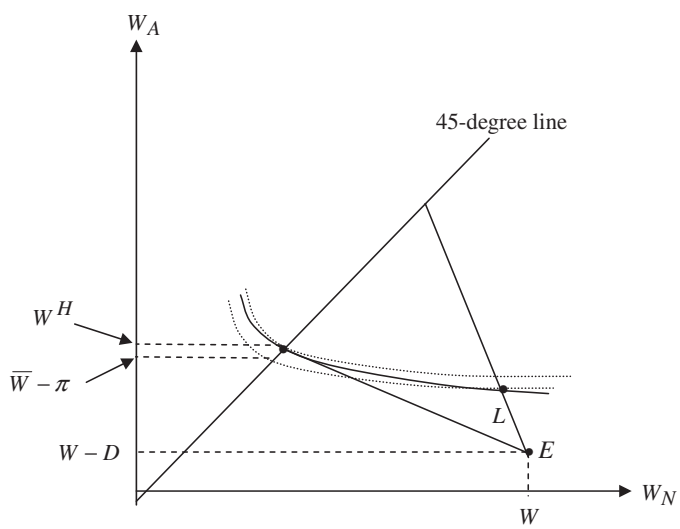

Figure 4. Deductible effect

insured wealth position $W^{H}{ }^{8}$ As $\bar{W}$ increases, the $H$-type indifference curves for $V$ cut the $L$-type fair-odds line above $L$, leading to the deductible effect. With DARA, $V$ becomes less risk averse as wealth increases, so the move from $\bar{W}-\pi$ to $W^{H}$ results in an acceptance set for $H$-types that contains the acceptance set that results when $V$ exhibits CARA. As a consequence, the indifference curve for $V\left(W^{H}\right)$ cuts the $L$-type fair-odds line at a point closer to $L$ when $V$ exhibits DARA rather than CARA, and the decline in the $L$-type deductible is smaller.

\section{The effect of background risk on the performance of insurance markets}

Our motivation in considering the effect of increased risk aversion on the critical value $\lambda^{*}$ is to determine the effect of background risk on the performance of insurance markets when insurance applicants are risk vulnerable. To that end, we assume that all insurance applicants are exposed to identical, uninsurable background risks that may or may not be correlated across applicants, but are assumed to be independent of the insurable,

${ }^{8}$ Note that $\bar{W}=\left(1-p^{H}\right) W_{N}^{L}+p^{H} W_{A}^{L}$ depicted in Figure 4 is calculated with respect to the original RS $L$-type contract, while the risk premium $\pi$ is associated with the more risk averse utility function $V$. As discussed in the proof of Lemma 2, an incremental increase in risk aversion causes $\bar{W}-\pi$ to fall below $W^{H}$, as depicted in the Figure, since the risk premium increases. In order to re-establish $\bar{W}-\pi=W^{H}, \bar{W}$ must increase, leading to a lower deductible in the $L$-type RS contract. 
foreground risks. As our results shall encompass unfair as well as fair background risks, DARA is a necessary condition for risk vulnerability as shown by Gollier and Pratt. Thus, Proposition 1 applies and we conclude that background risk can alter the nature of equilibrium by increasing $\lambda^{*}$ to a higher value denoted by $\lambda_{B R}^{*}$, causing the equilibrium to switch from the RS contracts to the WMS outcome. Moreover, even when the nature of equilibrium is unaffected, the equilibrium configuration of contracts is affected by background risk as the $H$-type applicants' acceptance sets become smaller.

We can distinguish three mutually exclusive and collectively exhaustive cases that differ with respect to the proportion of $H$-types in the applicant pool. $^{9}$

(a) $\lambda^{*}<\lambda_{B R}^{*} \leqslant \lambda$. In this case, equilibrium coincides with the $\mathrm{RS}$ contracts whether applicants are exposed to background risk or not. The $H$-types always receive full and fair insurance for the foreground risk, so their contract is unaffected by background risk. However, the $H$-types have a smaller acceptance set, so $L$-types bear a smaller deductible in their contract. As a consequence, background risk improves the insurance market's performance by lowering the signaling cost borne by $L$-types.

(b) $\lambda^{*} \leqslant \lambda<\lambda_{B R^{*}}$. In this case, the equilibrium coincides with the RS contracts in the absence of background risk, but switches to a Pareto-preferred, crosssubsidized pair when background risk is present. This change in the nature of equilibrium reduces the premium paid by $H$-types for full coverage, and results in a lower deductible for $L$-types, reducing the adverse selection cost they bear. Hence, background risk again improves the insurance market's performance, providing increased coverage of the insurable foreground risk.

(c) $\lambda<\lambda^{*}<\lambda_{B R}^{*}$. In this case, the equilibrium is a cross-subsidized separating pair of WMS contracts whether applicants are exposed to background risk or not. In the absence of background risk, the WMS outcome is illustrated in Figure 2 as the pair $(M, Y)$. Observe that $Y$ is a point of tangency between the locus $F L$ and the $L$-type indifference curve $U^{L}$, since $Y$ is the point on $F L$ most preferred by $L$-types. Hence, the pair $(M, Y)$ corresponds to the RS outcome relative to the endowment point labeled $E^{\prime}$ in Figure 5, and therefore this configuration is the same as that shown in Figure 1 with $E^{\prime}$ playing the role of $E, M$ playing the role of $H^{*}$, and $Y$ playing the role of $L$. It follows that the introduction of fair or unfair background risk creates

\footnotetext{
${ }^{9}$ Note that a fourth case - cross-subsidized contracts in the absence of background risk and breakeven contracts in its presence - is not possible because of Proposition 1 .
} 


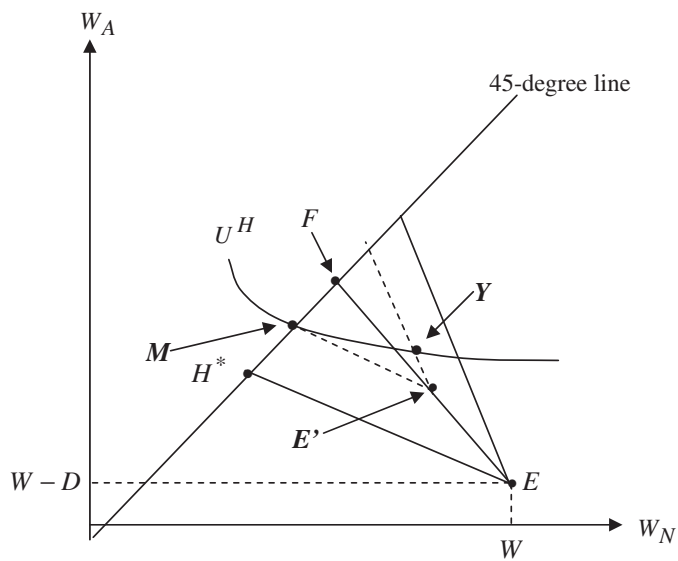

Figure 5. $\lambda<\lambda^{*}<\lambda_{B R^{*}}$

profitable, Pareto preferred defections from $(M, Y) .{ }^{10}$ Hence, the introduction of these background risks leads to an increase in coverage for $L$-types, as well as a reduction in the premium paid by $H$-types for full coverage. Once again, background risk improves the performance of insurance markets by reducing the signaling cost borne by $L$-types.

Thus, in each case, the insurance market affords better coverage of the foreground risk when applicants are exposed to fair or unfair background risk. However, since insurance applicants are risk averse, they are all made worse off by their individual exposures to fair or unfair background risk. In the remainder of this section, we investigate whether in any of the three cases the improved performance of the insurance market is sufficient for expected

${ }^{10}$ The result of Proposition 1 is that, for any initial endowment, $\lambda_{B R}^{*}<\lambda^{*}$. The critical values noted in the statement of case $\mathrm{C}\left(\lambda<\lambda<\lambda_{B R}^{*}\right)$ are defined relative to the initial endowment depicted as $E$ in the figures. Note, however, that $Y$ is the allocation on the locus $F L$ most preferred by $L$-types and so $\lambda$ is just equal to a new critical value $\lambda^{*}$ defined relative to the endowment $E^{\prime}$. Therefore, from Proposition 1, we know that the introduction of background risk increases this critical value, and so after the introduction of background risk there must exist profitable Pareto-preferred defections from $(M, Y)$. This situation is exactly analogous to the effect of $\lambda$ falling below $\lambda^{*}$ giving rise to profitable Pareto-preferred defections from the RS contracts, the case illustrated in Figure 2. The result is a lower premium for full coverage in the $H$-type contract and a lower deductible in the $L$-type contract. Finally, note that the movement from $E$ to $E^{\prime}$ could be achieved by either a zero-profit minimum coverage requirement (Dahlby, 1981) or by a balanced-budget tax and subsidy scheme (Crocker and Snow, 1985b). 
utilities to be higher in the presence of background risk. Put differently, the question is whether the increase in expected utilities caused by the improvement in the performance of the insurance market is sufficient to counteract the decrease in expected utilities caused by the insureds' exposures to background risk.

To explore this possibility, we introduce background risk by adding to the wealth of each insurance applicant a random variable $\delta \varepsilon$, where $\delta \in[0,1]$ is a scalar, and we examine the effect of increasing $\delta$. We assume that $\varepsilon$ has the same probability distribution for each applicant and is independent of the foreground risks, but make no assumption concerning the correlation of these risks across applicants. The critical assumption is that $\varepsilon$ represents an exogenous, uninsurable background risk with nonpositive expected value.

Note that background risk is introduced when $\delta$ rises above zero, and thereafter increasing $\delta$ induces a second-order stochastic dominance (SSD) deterioration of background risk. We therefore assume that utility is risk vulnerable in the stronger sense of Eeckhoudt et al. (1996), so that aversion to bearing the foreground insurable risk increases with SSD deteriorations of the background risk. In the following propositions, we show that, in each of the three cases, introducing or increasing background risk reduces the welfare of both risk types.

We begin with case $\mathrm{A}$, in which the equilibrium is always the separating pair of RS contracts. When applicants are exposed to background risk, the $H$-types are worse off since their contract is unaffected, but they must bear the cost of being exposed to an additional, independent risk. On the other hand, $H$-types have a smaller acceptance set and, as a consequence, the $L$-type contract has a lower deductible. Despite this improvement in the market's performance, we show that $L$-types are nonetheless worse off when a background risk is introduced or increased.

Proposition 2 In case A, introducing or increasing any fair or unfair background risk reduces the expected utility of both risk types.

Proof Let $W^{H}(\delta)$ and $\left[W_{N}^{L}(\delta), W_{A}^{L}(\delta)\right]$ denote the RS wealth positions for $H$ and $L$-types, respectively, given the background risk $\delta \varepsilon$. Since, in this case, the $H$-types always receive full and fair insurance for the foreground risk, $d W^{H}(\delta) /$ $d \delta=0$ and they are necessarily worse off when any fair or unfair background risk is introduced or increased. The $L$-type contract continues to earn zero profit as $\delta$ increases, so

$$
d W_{N}^{L} / d \delta=-\left[p^{L} /\left(1-p^{L}\right)\right]\left(d W_{A}^{L} / d \delta\right) .
$$


Using this relation, we find that the effect of an increase in $\delta$ on the expected utility of $L$-types is given by

$$
\begin{aligned}
d E\left[\left(1-p^{L}\right) U_{N}+p^{L} U_{A}\right] / d \delta= & \left(1-p^{L}\right) E\left[U_{N}^{\prime} \varepsilon\right] \\
& +p^{L} E\left[U_{A}^{\prime} \varepsilon\right] \\
& +p^{L}\left(E\left[U_{A}^{\prime}\right]-E\left[U_{N}^{\prime}\right]\right) \\
& \times\left(d W_{A}^{L} / d \delta\right),
\end{aligned}
$$

where the expectation is taken with respect to the random variable $\varepsilon$. Maintaining the incentive constraint for $H$-types,

$$
\begin{aligned}
E\left[U\left(W^{H}(\delta)+\delta \varepsilon\right)\right]= & \left(1-p^{H}\right) E\left[U\left(W_{N}^{L}(\delta)+\delta \varepsilon\right)\right] \\
& +p^{H} E\left[U\left(W_{A}^{L}(\delta)+\delta \varepsilon\right)\right],
\end{aligned}
$$

requires

$$
\begin{aligned}
- & E\left[\left(U_{H}^{\prime}-\left(1-p^{H}\right) U_{N}^{\prime}-p^{H} U_{A}^{\prime}\right) \varepsilon\right] \\
& +\left[\left(1-p^{H}\right) E\left[U_{N}^{\prime}\right] \frac{-p^{L}}{1-p^{L}}+p^{H} E\left[U_{A}^{\prime}\right]\right] \\
& \times\left(d W_{A}^{L} / d \delta\right)=0,
\end{aligned}
$$

which also uses (23).

Solving Eq. (26) for $d W_{A}^{L} / d \delta$ and substituting the result into Eq. (24), we find that the expected utility of $L$-types declines as $\delta$ increases if and only if

$$
\begin{aligned}
(1 & \left.-p^{L}\right) E\left[U_{N}^{\prime} \varepsilon\right]+p^{L} E\left[U_{A}^{\prime} \varepsilon\right] \\
& +p^{L}\left(E\left[U_{A}^{\prime}\right]-E\left[U_{N}^{\prime}\right]\right) \\
& \times \frac{E\left[\left(U_{H}^{\prime}-\left(1-p^{H}\right) U_{N}^{\prime}-p^{H} U_{A}^{\prime}\right) \varepsilon\right]}{p^{H}\left\{E\left[U_{A}^{\prime}\right]-\left[p^{L}\left(1-p^{H}\right) / p^{H}\left(1-p^{L}\right] E\left[U_{N}^{\prime}\right]\right\}\right.}<0 .
\end{aligned}
$$

Since $E\left[U_{A}{ }^{\prime}\right]>E\left[U_{N}{ }^{\prime}\right]$ and $p^{H}>p^{L}$, the denominator in the second expression is positive. Therefore, after multiplying by the denominator and rearranging terms, we find that inequality (27) is equivalent to

$$
\begin{aligned}
& \left(p^{H}-p^{L}\right)\left\{E\left[U_{N}^{\prime} \varepsilon\right] E\left[U_{A}^{\prime}\right]+\frac{p^{L}}{1-p^{L}} E\left[U_{A}^{\prime} \varepsilon\right] E\left[U_{N}^{\prime}\right]\right\} \\
& +p^{L}\left\{E\left[U_{A}^{\prime}\right]-E\left[U_{N}^{\prime}\right]\right\} E\left[U_{H}^{\prime} \varepsilon\right]<0 .
\end{aligned}
$$

Given risk aversion and our assumption that $E[\varepsilon] \leqslant 0, E\left[U_{s}^{\prime} \varepsilon\right]$ is negative for each $s \in\{N, A, H\}$. Hence, the inequality holds and we conclude that the decline in the deductible is insufficient to compensate the $L$-types for the cost of bearing the increase in background risk. 
The driving force behind this result comes from the incentive constraint. The $H$-types, with their full and fair insurance contract addressing the foreground risk, are worse off when background risk increases, and the incentive constraint ensures that they are equally worse off with the $L$-type RS contract. Hence, the decline in the $L$-type deductible must be too small to compensate for the increased cost of bearing background risk, and this effect carries over to the $L$-types.

Turning next to case $\mathrm{C}$, the equilibrium is always a cross-subsidized separating pair of WMS contracts.

Proposition 3 In case C, introducing or increasing any fair or unfair background risk reduces the expected utility of both risk types.

Proof Let $W^{H}(\delta)$ and $\left[W_{N}^{L}(\delta), W_{A}^{L}(\delta)\right]$ denote the cross-subsidized separating pair most preferred by $L$-types given $\delta$. For every value of $\delta \geqslant 0$, these pairs satisfy the incentive constraint (25) and, from (3), the zero-profit condition

$$
\begin{aligned}
& (1-\lambda)\left[\left(1-p^{L}\right) W_{N}^{L}(\delta)+p^{L} W_{A}^{L}(\delta)\right]+\lambda W^{H}(\delta) \\
& \quad=W-\left[(1-\lambda) p^{L}+\lambda p^{H}\right] D
\end{aligned}
$$

while maximizing the expected utility of $L$-types.

To examine the effect on expected utilities as $\delta$ increases, we first differentiate the zero-profit condition (29) with respect to $\delta$, solve for $d W^{H} / d \delta$, and then substitute the result into the differential equation implied by incentive constraint (25) and rearrange terms to obtain

$$
d W_{N}^{L} / d \delta=\left(T / T_{1}\right)-\left(T_{2} / T_{1}\right)\left(d W_{A}^{L} / d \delta\right)
$$

where

$$
\begin{aligned}
T & =E\left[\left(U_{H}^{\prime}-\left(1-p^{H}\right) U_{N}^{\prime}-p^{H} U_{A}^{\prime}\right) \varepsilon\right], \\
T_{1} & =\left(1-p^{H}\right) E\left[U_{N}^{\prime}\right]+\left(1-p^{L}\right)(1-\lambda) E\left[U_{H}^{\prime}\right] / \lambda, \text { and } \\
T_{2} & =p^{H} E\left[U_{A}^{\prime}\right]+p^{L}(1-\lambda) E\left[U_{H}^{\prime}\right] / \lambda .
\end{aligned}
$$

The effect on the expected utility of $L$-types is then given by

$$
\begin{aligned}
d E\left[\left(1-p^{L}\right) U_{N}+p^{L} U_{A}\right] / d \delta= & \left(1-p^{L}\right) E\left[U_{N}^{\prime} \varepsilon\right] \\
& +p^{L} E\left[U_{A}^{\prime} \varepsilon\right]+\left(T / T_{1}\right) \\
& \times\left(1-p^{L}\right) E\left[U_{N}^{\prime}\right] \\
& +\left(1 / T_{1}\right)\left[p^{L} T_{1} E\left[U_{A}^{\prime}\right]\right. \\
& \left.-\left(1-p^{L}\right) T_{2} E\left[U_{N}^{\prime}\right]\right] \\
& \times\left(d W_{A}^{L} / d \delta\right) .
\end{aligned}
$$


156

Observe first that the expected utility of $L$-types being maximized for a given value of $\delta$ implies that the $L$-type contract equates their marginal rate of substitution with the slope of a locus analogous to $F L$. This slope can be obtained from Eq. (4) by replacing $U_{s}^{\prime}$ with $E\left[U_{s}^{\prime}\right]$ for $s \in\{N, A, H\}$. The resulting expression is equal to $T_{1} / T_{2}$. Equating this ratio with the marginal rate of substitution, $\left(1-p^{L}\right) E\left[U_{N}{ }^{\prime}\right] / p^{L} E\left[U_{A}{ }^{\prime}\right]$, we find that the term in brackets on the third line of (32) vanishes.

Next, observe that $T$ is an increasing function of the probability $p^{H}$ under the assumption that $U$ exhibits DARA, that is,

$$
\begin{aligned}
\partial T / \partial p^{H} & =-\partial E\left[\left(\left(1-p^{H}\right) U_{N}^{\prime}+p^{H} U_{A}^{\prime}\right) \varepsilon\right] / \partial p^{H} \\
& =E\left[\left(U_{N}^{\prime}-U_{A}^{\prime}\right) \varepsilon\right] \\
& =\operatorname{cov}\left(U_{N}^{\prime}-U_{A}^{\prime}, \varepsilon\right)+E\left[U_{N}^{\prime}-U_{A}^{\prime}\right] E[\varepsilon]>0 .
\end{aligned}
$$

The inequality relies on the fact that $U$ is prudent, that is we have $U^{\prime \prime \prime}>0$, since prudence is implied by DARA, so that $U_{N}^{\prime \prime}$ exceeds $U_{A}^{\prime \prime}$, implying that $\operatorname{cov}\left(U_{N}^{\prime}-U_{A}^{\prime}, \varepsilon\right)$ is positive. Hence, $\partial T / \partial p^{H}$ is positive even if $E[\varepsilon]=0$. It follows that $T$ becomes smaller when $p^{L}$ replaces $p^{H}$, so that, with $T_{1}>0$, the righthand side of (32) is less than

$$
\begin{aligned}
& {\left[\left(1-p^{L}\right) E\left[U_{N}^{\prime} \varepsilon\right]+p^{L} E\left[U_{A}^{\prime} \varepsilon\right]\right]\left[1-\frac{\left(1-p^{L}\right) E\left[U_{N}^{\prime}\right]}{T_{1}}\right]} \\
& \quad+E\left[U_{H}^{\prime} \varepsilon\right] \frac{\left(1-p^{L}\right) E\left[U_{N}^{\prime}\right]}{T_{1}} .
\end{aligned}
$$

Recall that $E\left[U_{s}^{\prime} \varepsilon\right]$ is negative for each $s \in\{N, A, H\}$, given risk aversion and $E[\varepsilon] \leqslant 0$. Hence, the entire expression at (34) is negative, and the expected utility of $L$-types declines as $\delta$ increases, if the second term within brackets is positive.

To establish that this is the case, recall that, given $\delta$, the expected utility of $L$-types is maximized subject to the incentive constraint (25) and the zero-profit condition (29). The first-order conditions for the optimal choices of $W_{N}^{L}, W_{A}^{L}$, and $W^{H}$ given $\delta$ are

$$
\begin{gathered}
\left(1-p^{L}\right) E\left[U_{N}^{\prime}\right]-\mu\left(1-p^{H}\right) E\left[U_{N}^{\prime}\right] \\
-\gamma(1-\lambda)\left(1-p^{L}\right)=0, \\
p^{L} E\left[U_{A}^{\prime}\right]-\mu p^{H} E\left[U_{A}^{\prime}\right]-\gamma(1-\lambda) p^{L}=0, \quad \text { and } \\
\mu E\left[U_{H}^{\prime}\right]-\gamma \lambda=0,
\end{gathered}
$$


respectively, where $\mu$ and $\gamma$ are the Lagrange multipliers for the incentive constraint (25) and the zero-profit condition (29). Solving (37) for $\gamma$, substituting the result into (35), and solving for $\mu$, we obtain

$$
\mu=\frac{\left(1-p^{L}\right) E\left[U_{N}^{\prime}\right]}{T_{1}} .
$$

We conclude that (34) is negative as desired if $\mu$ is less than one. Again solving (37) for $\gamma$, substituting the result into (36), and solving for $\mu$ yields

$$
\mu=\frac{p^{L} E\left[U_{A}^{\prime}\right]}{T_{2}},
$$

which is indeed less than one. Hence, the expected utility of $L$-types declines as $\delta$ increases.

To determine the effect of background risk on the expected utility of $H$-types, we modify the problem of choosing $W_{N}^{L}, W_{A}^{L}$, and $W^{H}$ to maximize the expected utility of $L$-types by replacing the incentive constraint (25) with the utility constraint

$$
E\left[U\left(W^{H}+\delta \varepsilon\right)\right] \geq E\left[U\left(W^{H}(\delta)+\delta \varepsilon\right)\right] .
$$

Notice that this constraint requires that $H$-types be at least as well off at the contract solving the modified problem $\left(W^{H}\right)$ as they are in the solution to the original problem $\left(W^{H}(\delta)\right)$. Note as well that, in the modified problem, introducing any slack in either the zero-profit condition (29) or the utility constraint (40) would reduce the expected utility of the $L$-types below the value obtained in the original problem. As a consequence, both the original and the modified problems have the same solution.

To simplify the notation, let $U^{L}(\delta)$ and $U^{H}(\delta)$ denote the solution values for the expected utilities of $L$ - and $H$-types, and let $U^{H L}(\delta)$ denote the expected utility of $H$-types when they take the $L$-type contract. ${ }^{11}$ Applying the envelope theorem to the original problem, we find that the effect of an increase in $\delta$ on $L$-type expected utility is given by

$$
d U^{L} / d \delta=U_{\delta}^{L}+\mu\left(U_{\delta}^{H}-U_{\delta}^{H L}\right),
$$

where, as before, $\mu$ is the Lagrange multiplier associated with the incentive constraint (25), and the subscript $\delta$ denotes the partial derivative with respect

${ }^{11}$ More precisely, $U^{L}(\delta) \equiv\left(1-p^{L}\right) E\left[U\left(W_{N}^{L}(\delta)+\delta \varepsilon\right)\right]+p^{L} E\left[U\left(W_{A}^{L}(\delta)+\delta \varepsilon\right)\right], U^{H}(\delta)$ is given by the left-hand side of (25), and $U^{H L}(\delta)$ is given by the right-hand side when evaluated at the solution values of the appropriate (original or modified) problem. 
to $\delta$. Doing the same for the modified problem, we obtain

$$
d U^{L} / d \delta=U_{\delta}^{L}+\hat{\mu}\left[U_{\delta}^{H}-\left(d U^{H} / d \delta\right)\right],
$$

where $\hat{\mu}$ is the Lagrange multiplier for the utility constraint (40). Combining (41) and (42), we arrive at

$$
\hat{\mu} d U^{H} / d \delta=(\hat{\mu}-\mu) U_{\delta}^{H}+\mu U_{\delta}^{H L} .
$$

Since the partial derivatives are negative, we conclude that the expected utility of $H$-types declines as $\delta$ increases if $\hat{\mu}$ exceeds $\mu$.

To determine the value of $\hat{\mu}$, we use the first-order conditions for the optimal choices of $W_{N}^{L}$ and $W^{H}$ in the modified problem,

$$
\begin{gathered}
\left(1-p^{L}\right) E\left[U_{N}^{\prime}\right]-\hat{\gamma}(1-\lambda)\left(1-p^{L}\right)=0 \quad \text { and } \\
\hat{\mu} E\left[U_{H}^{\prime}\right]-\hat{\gamma} \lambda=0,
\end{gathered}
$$

where $\hat{\gamma}$ is the Lagrange multiplier for the zero-profit condition in the modified problem. Solving (45) for $\hat{\gamma}$, substituting the result into (43), and solving for $\hat{\mu}$, we obtain

$$
\hat{\mu}=\frac{\lambda E\left[U_{N}^{\prime}\right]}{(1-\lambda) E\left[U_{H}^{\prime}\right]} .
$$

Subtracting (38) from (46) and rearranging terms using (31), we arrive at

$$
\hat{\mu}-\mu=\frac{\lambda}{1-\lambda}\left[\frac{E\left[U_{N}^{\prime}\right]}{E\left[U_{H}^{\prime}\right]}\right] \frac{\left(1-p^{H}\right) E\left[U_{N}^{\prime}\right]}{T_{1}},
$$

which is positive, implying that the expected utility of $H$-types declines as $\delta$ increases.

Our final result concerns case $\mathrm{B}$, in which background risk causes the equilibrium to switch from the RS separating pair of contracts that breakeven individually to the WMS cross-subsidized separating pair.

Proposition 4 In case B, introducing or increasing any fair or unfair background risk reduces the expected utility of both risk types.

Proof We again add $\delta \varepsilon$ to the wealth of each insurance applicant and consider the effect of increasing $\delta$. The conclusion of the Proposition is then an immediate consequence of Propositions 2 and 3. If $\lambda=\lambda^{*}$, then the market is in the knife-edge situation depicted in Figure 1 prior to the introduction of background risk. Hence, this situation is effectively covered by Proposition 3 inasmuch as the equilibrium $L$-type contract for all $\delta>0$ is the one most 
preferred by $L$-types among those that satisfy the incentive compatibility constraint (25) and the zero-profit condition (29). Turning to situations in which $\lambda>\lambda^{*}$, the initial equilibrium (corresponding to $\delta=0$ ) is the RS pair, and these contracts continue to constitute the equilibrium as $\delta$ increases until a critical value $\delta^{*}$ is reached at which $\lambda_{B R}^{*}=\lambda$. Thus, the analysis of Proposition 2 for case A applies when $\delta$ is less than $\delta^{*}$, and thereafter the analysis of Proposition 3 for case $\mathrm{C}$ applies. Thus, for case $\mathrm{B}$, we conclude that introducing or increasing any fair or unfair background risk reduces the expected utility of both risk types.

\section{Conclusions}

Insurance applicants may be exposed to uninsurable background risks that increase their aversion toward bearing the foreground, insurable risks. As a result, background risk can influence the performance of competitive insurance markets that must also deal with adverse selection by altering incentive compatibility constraints as well as the attractiveness of competing contractual offers. We show that, when insurance applicants are risk vulnerable with respect to both introductions and SSD deteriorations of fair or unfair background risk, the separating RS equilibrium is attained over a narrower range of markets as a result of background risk. This works to the advantage of both high- and low-risk applicants when background risk results in the WMS equilibrium by reducing the premium high-risks pay for full coverage and reducing the deductible borne by low-risks. Even when the RS outcome is the equilibrium in the presence of background risk, the adverse selection externality is mitigated as the greater aversion of high-risks toward bearing the insurable risk relaxes the incentive constraint, thereby allowing for an expansion in the coverage afforded low-risks. Thus, background risk always enhances the performance of insurance markets under adverse selection.

Nonetheless, exposure to background risk is costly, and we find that for all risk types this cost outweighs the gains from the improved performance of markets insuring the foreground risk. Therefore, innovations that limit exposures to background risk or that extend insurance coverage to these risks would improve the welfare of all insurance applicants.

\section{References}

Arnott, R.J. and Stiglitz, J.E. (1988) 'Randomization with asymmetric information', Rand Journal of Economics 19 (Fall): 344-361.

Asheim, G.B. and Nilssen, T. (1996) 'Non-discriminating renegotiation in a competitive insurance market', European Economic Review 10 (December): 1717-1736. 
160

Crocker, K.J. and Snow, A. (1985a) 'The efficiency of competitive equilibria in insurance markets with asymmetric information', Journal of Public Economics 26 (March): 207-219.

Crocker, K.J. and Snow, A. (1985b) 'A simple tax structure for competitive equilibrium and redistribution in insurance markets with asymmetric information', Southern Economic Journal 51 (April): 1142-1150.

Dahlby, B.G. (1981) 'Adverse selection and pareto improvements through compulsory insurance', Public Choice 37: 547-558.

Eeckhoudt, L., Gollier, C. and Schlesinger, H. (1996) 'Changes in background risk and risk taking behavior', Econometrica 64 (May): 683-689.

Eeckhoudt, L. and Kimball, M. (1992) 'Background risk, prudence, and the demand for insurance', in G. Dionne (ed) Contributions to Insurance Economics, Boston: Kluwer Academic Publishers, pp. 239-254.

Gollier, C. and Pratt, J.W. (1996) 'Risk vulnerability and the tempering effect of background risk', Econometrica 64 (September): 1109-1123.

Hellwig, M. (1987) 'Some recent developments in the theory of competition in markets with adverse selection', European Economic Review 31 (February/March): 319-325.

Kimball, M.S. (1990) 'Precautionary saving in the small and in the large', Econometrica 58 (January): 53-73.

Landsberger, M. and Meilijson, I. (1994) 'Monopoly insurance under adverse selection', Journal of Economic Theory 63 (August): 392-407.

Landsberger, M. and Meilijson, I. (1999) 'A general model of insurance under adverse selection', Economic Theory 14 (August): 331-352.

Miyazaki, H. (1977) 'The rat race and internal labor markets', Bell Journal of Economics 8 (Autumn): 394-418.

Pratt, J. (1964) 'Risk aversion in the small and in the large', Econometrica 32: 122-136.

Rothschild, M. and Stiglitz, J.E. (1976) 'Equilibrium in insurance markets: An essay on the economics of imperfect information', Quarterly Journal of Economics 90 (November): 629-649.

Smart, M. (2000) 'Competitive insurance markets with two unobservables', International Economic Review 41 (February): 153-169.

Spence, M. (1978) 'Product differentiation and performance in insurance markets', Journal of Public Economics 10 (December): 427-447.

Wambach, A. (2000) 'Introducing heterogeneity in the Rothschild-Stiglitz model', Journal of Risk and Insurance 67 (December): 579-592.

Wilson, C.A. (1977) 'A model of insurance markets with asymmetric information', Journal of Economic Theory 16 (December): 167-207. 